\title{
APPRENDIMENTO E CAMBIAMENTO NELLE POLITICHE PUBBLICHE. IL RUOLO DELLE IDEE E DELLA CONOSCENZA
}

\author{
di Elisabetta Gualmini
}

\section{Introduzione}

Le più recenti direzioni di ricerca nell'ambito dell'analisi delle politiche pubbliche rivelano un interesse sempre più marcato verso lo studio delle componenti cognitive del fenomeno politico: le idee e gli assunti normativi che informano l'azione dei decisori pubblici ${ }^{1}$. Un'ampia letteratura, di prevalente origine anglosassone, si è sviluppata intorno al concetto di policy learning, inteso come processo di revisione degli obiettivi e degli strumenti contenuti nei programmi pubblici, nonché delle premesse di valore a cui essi rimandano, in risposta agli errori cumulati nell'esperienza passata, all'acquisizione di nuove informazioni e all'innescarsi di logiche imitative. Più in dettaglio, si individuano tre tematiche salienti intorno alle quali pare meritorio interrogare questa letteratura: la possibilità di spiegare il cambiamento nelle politiche pubbliche accostando alle variabili strutturali, prevalentemente riconducibili alla fenomenologia del potere e del conflitto, le dimensioni cognitive da cui esse non possono prescindere; il riconoscimento della rilevanza della conoscenza e della circolazione delle idee non solo come fonti di innovazione, ma come frames entro cui la formulazione stessa dei problemi di policy, la loro salienza e la loro trattabilità vengono costruite socialmente; l'accettazione dell'indetermina-

Desidero ringraziare Maurizio Ferrera, Giovan Francesco Lanzara e Stefano Zan per $i$ preziosi suggerimenti a una prima versione di questo lavoro. Ringrazio inoltre Giorgio Freddi e David Soskice per il supporto tecnico e scientifico ricevuto nell'ambito del Centro di Analisi delle Politiche Pubbliche di Bologna e del Wissenschaftszentrum für Sozialforschung di Berlino.

' In particolare, facciamo riferimento a Dobuzinskis (1992); Haas (1992); Hall (1993); Rosenau (1993); Sabatier e Jenkins-Smith (1993); Schneider e Ingram (1991, 1993); Schram (1993); Weiss (1989).

RIVISTA ITALIANA DI SCIENZA POLITICA / a. XXV, n. 2, agosto 1995 
tezza causale e dell'ambiguità strutturale nella descrizione (e spiegazione) del rapporto tra attori, problemi e soluzioni.

Non mancano tuttavia numerosi problemi metodologici connessi all' «importazione» del nuovo modello interpretativo, inerenti, da un lato, al tentativo di comprendere entro un'unica categoria (quella appunto dei processi di apprendimento) suggestioni teoriche eterogenee, con il rischio di incorrere in un eccessivo «stiramento del concetto»; e dall'altro, all'applicabilità delle dimensioni cognitive alla ricerca politologica. Molti contributi sull'apprendimento di policy risultano infatti eleganti esperimenti condotti in vitro, avulsi dalla complessità del fenomeno politico. La mancanza di ricerche empiriche, o meglio la «iperteorizzazione» del concetto (Bennett e Howlett 1992), provocata da una rincorsa affrettata verso le suggestioni delle scienze cognitive, non aiuta ad approfondire le nuove ipotesi.

Il presente contributo si propone dunque due obiettivi. Dapprima intendiamo rivedere criticamente la letteratura sull'apprendimento proponendone una prima sistematizzazione teorica. Convinti che «nessun sapere scientifico è mai nato senza aver ordinato e precisato il proprio vocabolario» (Sartori 1980, I), riteniamo che l'individuazione di chiare definizioni concettuali s'imponga come esigenza primaria. Successivamente affronteremo la vexata quaestio del rapporto tra apprendimento e cambiamento, illustrando come intorno a questa tematica sia possibile ravvisare significative aree d'intersezione con l'analisi politica.

L'ipotesi da cui muoviamo è che lo studio dei processi di apprendimento e del ruolo delle componenti cognitive dell'azione politica sia in grado di riorientare il dibattito sulle determinanti del cambiamento politico. Rivolgere l'attenzione ai processi di apprendimento significa non solo individuare nuove variabili per descrivere il percorso evolutivo delle politiche, ma, in una prospettiva più ampia, indagare intorno al modo in cui l'emergere di nuove soluzioni viene costantemente filtrato attraverso le percezioni e le interpretazioni soggettive degli attori.

\section{Gli studi sul policy learning: una prima sistematizzazione}

È possibile ordinare l'eterogenea produzione relativa al policy learning in cinque distinte «teorie»² (Gualmini 1995), basate su

${ }^{2}$ Il termine «teoria» viene qui inteso in un'accezione lata come insieme di ipotesi 
una diversa nozione di apprendimento, in cui si rintracciano sollecitazioni provenienti da vari settori disciplinari (fig. 1). I cinque modelli si situano all'interno di un continuum alle cui estremità s'individuano due opposte definizioni di apprendimento: apprendimento come valutazione (evaluation-oriented learning) e apprendimento come processo generatore di conoscenza (knowledge-oriented learning) ${ }^{3}$. Al primo polo si avvicinano gli autori che utilizzano un'accezione strumentale (non di rado prescrittiva) del termine collegandolo alla valutazione dell'impatto e delle conseguenze dei programmi pubblici rispetto agli obiettivi attesi. La misurazione dello scarto tra risultati effettivi e scopi prefissati permette di rilevare le devianze e fornisce indicazioni normative sul come le politiche dovrebbero essere attuate. Tale concezione affonda le proprie radici in un paradigma teleologico, che fa del raggiungimento degli obiettivi (e quindi dell'efficienza) l'oggetto privilegiato dell'apprendimento, sullo sfondo di un diffuso ottimismo tecnocratico. Vicino al secondo polo sono compresi, invece, gli approcci che privilegiano un uso cognitivo-epistemico di apprendimento, inteso come un processo generatore di conoscenza. L'acquisizione di nuove informazioni e la diffusione di nuove idee provocano slittamenti nel contesto in cui prendono vita le politiche che incentivano la sperimentazione di nuovi programmi. Le dinamiche cognitive, $i$ valori, i sistemi di credenza che informano l'azione dei decisori pubblici diventano rilevanti per sé. Le politiche sono «fenomeni normativi» (Hall 1986; Pappalardo 1991 e 1992) con un duplice ruolo: fonte di riconoscimento e di stabilità e, al contempo, campo mutevole di azione e di innovazione. Si sfuma il rapporto di determinazione causale tra attori, problemi e soluzioni; le politiche trascendono gli obiettivi, che assumono una valenza simbolica diventando sfuocati punti di riferimento (Bateson 1976). Dall'interesse sull'output, l'attenzione viene spostata, in maniera quasi autoreferenziale, sul processo stesso. «Dare un senso a ciò che accade» diventa l'essenza del policy making (Regonini 1993).

interpretative di ausilio alla descrizione dei fenomeni sociali e non, in senso neopositivista, come insieme di proposizioni collegate gerarchicamente (Hempel 1968), verificabili secondo la logica del metodo sperimentale. Essa diventa dunque sostanzialmente intercambiabile con il termine «modello».

${ }^{3} \grave{E}$ opinione condivisa che le teorie dell'apprendimento possano essere distinte sulla base del grado di adattamento o, viceversa, di elaborazione attiva richiesta ai soggetti nei confronti degli stimoli esterni. Si vedano Gherardi (1994) e Lanzara (1993; 1994). 
Evaluation-oriented learning

Knowledge-oriented learning

\begin{tabular}{|c|c|c|c|c|}
\hline $\begin{array}{l}\text { Apprendimento } \\
\text { come correzione } \\
\text { degli errori }\end{array}$ & $\begin{array}{l}\text { Apprendimento } \\
\text { come risposta a } \\
\text { stimoli esterni }\end{array}$ & $\begin{array}{l}\text { Apprendimento } \\
\text { come imitazione } \\
\text { e diffusione di } \\
\text { programmi }\end{array}$ & $\begin{array}{l}\text { Apprendimento } \\
\text { come } \\
\text { cambiamento dei } \\
\text { sistemi di credenza }\end{array}$ & $\begin{array}{l}\text { Apprendimento } \\
\text { come processo } \\
\text { generatore di } \\
\text { conoscenza }\end{array}$ \\
\hline $\begin{array}{l}\text { - Studi sull'implementazio- } \\
\text { ne [Wildavsky, Majone] } \\
\text { - Teoria dell'errore e studi } \\
\text { sui processi decisionali } \\
\text { [Landau, Simon, } \\
\text { Lindblom] }\end{array}$ & $\begin{array}{l}\text { - Comparative Public } \\
\text { Policy [Heclo] } \\
\text { - Cibernetica e analisi dei } \\
\text { sistemi [Deutsch, Easton] }\end{array}$ & $\begin{array}{l}\text { - Lesson drawing [Rose] } \\
\text { - Epistemic Communities } \\
\text { [Haas] }\end{array}$ & $\begin{array}{l}\text { - Advocacy coalition } \\
\text { framework [Sabatier e } \\
\text { Jenkins-Smith } \\
\text { - Policy Paradigm [Hall] } \\
\text { - Organizational Learning } \\
\text { [Argyris e Schön] }\end{array}$ & $\begin{array}{l}\text { - Metafore post-moderne } \\
\text { del policy making e } \\
\text { cibernetica di secondo } \\
\text { ordine [Dobuzinskis, } \\
\text { Schram, Schneider] } \\
\text { - Teorie della complessità } \\
\text { [Maturana e Varela] }\end{array}$ \\
\hline
\end{tabular}

FIG. 1. Quadro sinottico degli approcci al policy learning e tradizioni di ricerca da cui sono originati. 
Tra i due poli del continuum si collocano i modelli che esprimono posizioni intermedie delineando passaggi sequenziali dalle teorie adattivo-comportamentali alle teorie cognitive.

Analizziamo ora i cinque modelli più in dettaglio. La possibilità di imparare correggendo gli errori verificatisi nell'implementazione dei programmi pubblici viene accolta da Wildavsky come finalità principale dell'analisi delle politiche. L'accezione prescrittiva deriva dalla stretta correlazione tra apprendimento politico e possibilità di risolvere problemi (quindi di migliorare le politiche). «... Per gli analisti, la presenza di problemi implica una reale possibilità di soluzione, in quanto non vi sarebbe alcuna analisi delle politiche se non vi fosse alcuna azione da raccomandare. L'analisi, in cui le soluzioni ci indicano se abbiamo problemi, implica l'apprendimento di ciò che si è costretti a preferire attraverso l'esclusione di ciò che non si può ottenere. Se non esistessero ostacoli alla realizzazione degli obiettivi, nessuna analisi avrebbe ragione d'essere» $(1979,42)$. Riflettendo su alcuni casi di fallimento dei programmi lanciati negli anni sessanta e settanta (educazione, sanità, criminalità), lo studioso dimostra come le agenzie governative sostituiscano sovente agli scopi iniziali obiettivi maggiormente raggiungibili. La ritirata strategica dagli obiettivi diventa un'operazione razionale in un processo politico amministrativo che procede per approssimazioni successive. L'apprendimento scaturisce dalla graduale riduzione dello scarto tra mezzi e fini, attraverso la ridefinizione degli obiettivi, la sostituzione dei mezzi, o la riformulazione dei problemi. In senso popperiano, gli individui esperiscono la realtà grazie alla falsificazione delle proprie supposizioni ${ }^{4}$. Anche Majone suggerisce di pensare all'apprendimento come ad una «valutazione formativa» $(1987,34)$. L'attento monitoraggio del decorso dei programmi pubblici consente di prevenire e di neutralizzare le deviazioni. «[Ancora] più importante è imparare come i programmi vengono implementati, chi ci guadagna e chi ci perde, se il programma sta realizzando ciò che ci si prefigura$\mathrm{va}$, e, in caso contrario, come può essere migliorato oppure interrotto» $(1989,167)$. Questa concezione strutturata di apprendimento viene convincentemente integrata dalla rilevazione di

${ }^{4}$ Di qui la valenza creatrice dell'analisi, la possibilità di favorire attivamente le condizioni per l'apprendimento sociale e politico attraverso il miglioramento della qualità delle interazioni tra $\mathrm{i}$ cittadini, in curiosa sintonia con gli intenti della teoria critica habermasiana (Forester 1982). 
variabili soft: la persuasione, il discorso argomentativo, la retorica e il linguaggio diventano categorie fondamentali per lo studio del fenomeno politico che viene a configurarsi come il punto di intersezione tra le dimensioni proprie della conoscenza e le dimensioni proprie del potere e degli interessi.

La progettazione di istituzioni capaci di rilevare gli errori trova paralleli significativi nella teoria dell'errore e negli studi sui processi decisionali. Martin Landau auspica la progettazione di self-correcting organizations (1973); Crozier definisce le organizzazioni burocratiche come sistemi incapaci di correggere $\mathrm{i}$ propri errori e, a fortiori, incapaci di apprendere (1978). Gli studi sull'incrementalismo (Simon 1967; Braybrooke e Lindblom 1970) configurano il policy making come un processo incrementale che procede attraverso una sequenza di prove ed errori successivi. «La politica è diretta a un problema; viene provata, modificata, rimessa alla prova nella forma corretta, modificata di nuovo, e così di seguito. In breve, le politiche incrementali si susseguono le une dopo le altre nella soluzione di un dato problema» $(1970,73)^{5}$.

Una definizione diversa di apprendimento, di diretta derivazione comportamentista, viene fornita da Heclo: «un'alterazione del comportamento relativamente persistente nel tempo che risulta dall'esperienza; di solito questa alterazione è concettualizzata come un cambiamento in risposta a qualche stimolo percepito» $(1974,306)$. Il richiamo alla psicologia comportamentista non si ferma qui. Heclo distingue tra due tipi di apprendimento. Il primo, per riflesso condizionato, esprime la tendenza dell'individuo a riprodurre le medesime risposte istituzionali, già sperimentate e quindi considerate «naturali», anche in presenza di stimoli differenti; incrementalismo e aggiustamenti al margine sono i risultati principali. Il secondo, definito da un tipo di «condizionamento operativo o strumentale», introduce un cambiamento più profondo e innovativo, perché l'individuo tenendo conto dei vincoli e delle opportunità dell'ambiente circostante modifica di volta in volta il proprio comportamento in un contesto di maggiore adattabilità. La sapiente combinazione di teoria politica, ricerca storica e analisi delle politiche consente all'autore di inaugurare originali ragionamenti che muovono

5 Le politiche sono «rimediali, seriali, esplorative» (Braybrooke e Lindblom 1970, 74) e quindi refrattarie alla grande riforma. 
da una critica sostanziale agli studi tradizionali sulle politiche: la tendenza ad attribuire $\mathrm{i}$ cambiamenti nelle politiche pubbliche a mutamenti nelle relazioni di potere tra le diverse coalizioni degli interessi ${ }^{6}$. I cambiamenti nelle relazioni di potere (powering) (spostamenti nelle preferenze elettorali, ricambio dell'élite di governo e mobilitazione di nuovi gruppi) non si traducono simultaneamente in nuovi programmi. Essi vengono filtrati dalle percezioni e dalle cognizioni degli attori che adattano le proprie strategie sulla base di ciò che viene percepito conveniente, modificando e «reinventando» (Rice e Rogers 1980) gli stimoli originali. Il processo politico altro non è che un processo di apprendimento sociale espresso attraverso programmi. Il programma, più che configurarsi come l'esito di un processo decisionale razionale, è un campo di sperimentazione in continuo mutamento. È possibile individuare tre distinti livelli a cui si svolgono i processi di apprendimento. Il primo, definito sociale, concerne gli individui, considerati come agenti principali del cambiamento nella società. Il secondo è dato dal reticolo degli scambi interorganizzativi, costituito dalle organizzazioni di appartenenza: partiti, sindacati e istituzioni di governo. Il terzo livello di apprendimento è interno alla policy stessa: in questo caso sono le caratteristiche specifiche dei programmi, gli strumenti e i metodi adottati nel passato, così come vengono ereditati nel tempo, ad esercitare un impatto sullo sviluppo della politica favorendo la riedizione di sentieri già conosciuti e di logiche imitative. Pur liberando l'analisi del policy making dagli aspetti più meccanici (e prescrittivi) che caratterizzano gli studi precedenti, gettando così le fondamenta per un'analisi descrittiva ed esplicativa del processo politico verso cui gli approcci successivi sono largamente debitori, Heclo rimane ancorato ad una definizione deterministica di apprendimento che rivela forti legami con le interpretazioni sistemiche e funzionaliste (Deutsch $1963)^{7}$. Il «bias inputista» con cui l'autore guarda agli effetti di

${ }^{6}$ La citatissima combinazione tra powering e puzzling è tuttora illuminante. «Tradition teaches that politics is about conflict and power. (...) This is a blinkered view of politics and particularly blinding when applied to social policy. (...) Governments not only "power" (or whatever the verb form of that approach might be); they also puzzle. Policy making is a form of collective puzzlement on society behalf; it entails both deciding and knowing» (Heclo 1974, 304-305).

${ }^{7}$ In particolare, nei lavori di Heclo si scorge l'influenza di Deutsch (1963) e dei suoi tentativi di combinare l'analisi sistemica con la neonata scienza cibernetica. Al centro di questo filone si colloca la progettazione di sistemi flessibili capaci di processare informazioni in analogia con il funzionamento del cervello umano. Il concetto di feed- 
retroazione, considerati come servomeccanismi automatici e non come meccanismi propulsori del processo politico, ha spinto numerosi studiosi a potenziare questo ragionamento con altre ipotesi.

Una definizione agile e intuitiva di apprendimento è individuabile nel lesson drawing propostoci da Rose (1991), secondo il quale è possibile imparare imitando. I decision makers guardano agli esempi sviluppati in altri settori di policy oppure nel medesimo settore di policy in altri contesti e adottano programmi altrove considerati di successo. Muovendo da una situazione di insoddisfazione verso le politiche esistenti, si intraprende la ricerca di nuove soluzioni. In questa prospettiva trova spazio il concetto di comunità epistemica, definita come network di professionisti a cui vengono riconosciute esperienza e competenza in particolari settori di policy (Haas 1992, 3), in grado di favorire la circolazione nazionale e internazionale delle idee promuovendo campi di innovazione comuni. L'esempio più citato è quello delle politiche macro-economiche, che hanno registrato processi di imitazione internazionale tanto per la diffusione delle politiche keynesiane, quanto degli esperimenti di deregulation. La comunità epistemica può operare a livello locale, nazionale e internazionale. La condivisione di conoscenze tecniche e di comuni visioni del mondo (non l'affiliazione a gruppi o partiti) è il criterio di appartenenza. Il trasferimento dei programmi può avvenire attraverso cinque diverse modalità: per copiatura, per emulazione, per ibridazione, per sintesi, o infine per ispirazione ${ }^{8}$. In questo senso «il policy maker è un ingegnere sociale che cerca di sfruttare la conoscenza in modo strumentale» (Rose 1991, 5). La capacità di elaborare stimoli esistenti adattandoli a un contesto predefinito viene premiata rispetto alla progettazione di nuovi programmi. La diffusione delle idee da un'agenda all'altra produce effetti di feedback essenziali per il

back, come meccanismo di retroazione e di autoregolazione, destinato a fertilizzare numerosi settori disciplinari, viene concettualizzato in questo ambito.

${ }^{8}$ Con i termini «copiatura» ed «emulazione» l'autore fa riferimento all'adozione di programmi in vigore in altri settori; nel primo caso l'adozione è completa, mentre nel secondo i programmi subiscono modificazioni e aggiustamenti a seconda delle circostanze. I meccanismi della «ibridazione» e della «sintesi» indicano la combinazione, entro un unico programma, di elementi derivanti da programmi diversi; la sola differenza risiede nel maggior numero di stimoli originari richiesti dall'operazione di sintesi. L'«ispirazione», infine, viene definita come il processo attraverso il quale le politiche sviluppate in altri settori di policy o in altri paesi fungono da stimolo per la creazione di programmi del tutto innovativi rispetto alle fonti originarie (Rose 1991, 22). 
riorientamento consapevole del processo politico (Kingdon 1984; Schneider e Ingram 1991).

Un tentativo di alleviare il determinismo insito nella definizione di Heclo accentuandone la portata cognitiva viene compiuto da Sabatier e Jenkins-Smith, studiosi particolarmente attenti al filone dell'apprendimento organizzativo. Il policy-oriented learning viene identificato in «alterazioni relativamente durature delle intenzioni e dei comportamenti che risultano dall'esperienza e che riguardano il compimento o la revisione degli assunti normativi contenuti nei sistemi di credenza individuali e collettivi» (1993, 42). L'introduzione del concetto di sistema di credenza, l'attenzione alla base cognitiva dei comportamenti deliberati, sposta il processo da una dimensione esterna (apprendimento come risposta a stimoli esogeni) ad una dimensione interna (apprendimento come mutamento dei valori e delle preferenze endogene). Il cambiamento nelle politiche non è più solo attribuito alla modificazione delle strategie degli attori, ma ai cambiamenti nel retroterra cognitivo, nelle rappresentazioni che governano le strategie medesime. Un sistema di credenza, inteso come l'insieme degli assunti normativi e dei meccanismi causali che connettono valori a obiettivi, si articola in tre livelli. Il livello più profondo è costituito dal nocciolo normativo che contiene gli assunti fondamentali a cui le élites ancorano la loro visione del mondo, il secondo livello comprende le strategie d'azione attivate dagli individui per perseguire gli obiettivi, il terzo livello include gli aspetti secondari, le routine strumentali al perseguimento dei fini'.

Ancora a Heclo si ispira Peter Hall nella sua vasta produzione relativa allo studio della diffusione delle idee nel policy making (1986; 1993). Hall identifica due problemi nell'uso del concetto di apprendimento da parte di Heclo e di alcuni teorici dello stato che al lavoro di Heclo fanno riferimento (Weir e Skocpol 1985): la genericità nell'uso del concetto e la tendenza a restringerne gli spazi entro $i$ confini delle istituzioni formali ${ }^{10}$

${ }^{9}$ La struttura dei sistemi di credenza richiama da vicino la configurazione delle teorie dell'azione in Argyris e Schön (assunti normativi - strategie d'azione - obiettivi) e la distinzione tra apprendimento ad anello semplice (single-loop learning) e apprendimento ad anello doppio (double-loop learning) (1978).

${ }^{10} \mathrm{La}$ critica di Hall verso gli approcci stato-centrici potrebbe essere estesa ad autori come Etheredge e Short, che configurano l'apprendimento come processo eminentemente correlato alla funzione di governo (1983). L'apprendimento si riflette in un aumento di competenza nel trattamento dei problemi e di efficacia nella loro soluzione. 
(schiacciando il concetto di apprendimento sul concetto di autonomia dello stato). Sulla base di un'ampia ricerca sulle politiche macroeconomiche in Gran Bretagna dal 1970 al 1989, in particolare sulla sostituzione delle politiche keynesiane con politiche monetariste, Hall intende approfondire la natura dell'apprendimento, che viene definito come il processo attraverso cui vengono modificati gli obiettivi e le tecniche di policy sulla base dell'esperienza passata e dell'acquisizione di nuove informazioni. Riprendendo le categorie proposte da Bateson (1976), Hall identifica tre distinti livelli del processo. Nell'apprendimento di primo tipo solamente il contesto (le condizioni complessive degli strumenti a disposizione) subisce variazioni, lasciando inalterati gli strumenti stessi e gli obiettivi. Il riorientamento degli strumenti, insieme al contesto, avviene nel cambiamento di second'ordine; mentre il cambiamento di terzo tipo, più profondo e radicale, porta con sé una radicale trasformazione del contesto, delle tecniche, e dei fini perseguiti. In quest'ultimo caso si assiste alla sostituzione del «paradigma di policy» dominante, definito dall'insieme di simboli, di assunti e di valori contenuti nelle strategie d'azione e nelle pratiche di comunicazione attivate dai decisori pubblici. È forse questo il punto più innovativo: la sostituzione delle idee dominanti, in analogia con l'avvicendarsi dei paradigmi scientifici analizzati da Kuhn, genera nuova conoscenza e determina la modificazione dei frames di riferimento in cui è racchiusa la definizione delle norme formali e informali che regolano i processi politici. La sfera della metapolitica (Cotta 1994) è soggetta ad urti e scricchiolii che impongono il rimodellamento delle regole del gioco. Mentre i cambiamenti di primo e secondo tipo sono casi di policy making «ordinario» e corrispondono ai processi di apprendimento descritti da Heclo e riportati dal main stream della teoria dello stato, il cambiamento di terzo tipo implica la mobilitazione di un numero elevato di attori (gruppi, partiti, esperti, e liberi cittadini) e porta alla creazione di nuove rappresentazioni collettive e di nuovi modelli d'azione. Lo slittamento del paradigma di policy smentisce l'ipotesi di un processo di apprendimento ristretto ai confini dello Stato, allargandosi sino a comprendere tutti gli attori sociali e biforcando la linea Stato-società.

Le metafore post-moderne del policy making si spingono ancora oltre e si allontanano definitivamente da ogni paradigma strumentale. In assenza di chiare relazioni di causa ed effetto tra problemi e soluzioni e a fronte della crescente imprevedibi- 
lità dei fenomeni sociali, l'interesse della ricerca si sposta dagli obiettivi ai mezzi, alla logica di funzionamento del processo medesimo. L'analisi post-moderna intende riflettere su ciò che viene dato per scontato dai decisori pubblici nel processo politico (Rosenau 1993). La decostruzione dei significati e la dematerializzazione delle strutture porta al centro dell'attenzione il problema della ricerca dell'identità (che non è più considerata predefinita). Le politiche sono artefatti, «sistemi di significati simbolici» (Schram 1993) che servono a ridurre le pressioni sociali. Scompaiono le categorie del razionale e del non razionale per lasciare posto a dimensioni rarefatte dove il tempo e lo spazio non possono essere definiti a priori. L'incertezza, la discontinuità e l'indeterminatezza sono caratteristiche strutturali delle politiche; l'ambivalenza viene percepita come un vantaggio (Regonini 1994) che rende vana ogni ossessiva richiesta di control$\mathrm{lo}^{11}$. Il linguaggio e la comunicazione politica sono gli unici strumenti di costruzione e di interpretazione della realtà politica. Le politiche contribuiscono a creare la realtà con cui esse stesse si confrontano (Maturana e Varela 1987); in circoli autoreferenziali esse diventano causa di se stesse. L'analogia è con i sistemi autopoietici studiati dalla cibernetica di secondo ordine. Il concetto di apprendimento che queste teorie propongono è totalmente differente rispetto alle altre nozioni: esso viene identificato nel pensiero riflessivo (reflexive understanding: Dobuzinskis 1992, 368), nella capacità di riflettere sull'adeguatezza della propria identità e sulla coerenza della propria azione nel tempo. E un apprendimento di secondo ordine, un deutero-apprendimento, che implica la rimessa in discussione delle regole e delle norme che definiscono la nostra capacità di apprendere.

\section{Apprendimento e cambiamento}

Le teorie sul policy learning e sulle componenti cognitive dell'azione politica sono state utilizzate con intenti descrittivi ed

11 Secondo Regonini l'indeterminatezza dell'azione sociale conduce alla presa d'atto che «gli elementi che connotano una politica pubblica sono collegati non da catene di dipendenza, ma da relazioni di tipo configurativo, caratterizzate da due tratti: la compatibilità, che rende effettivamente riscontrabili solo alcune delle combinazioni in astratto possibili, e l'elasticità, che consente margini più o meno ampi per compensazioni e aggiustamenti tra i fattori, considerati tra loro interdipendenti» $(1994,180)$. 
esplicativi nell'interpretazione del cambiamento delle politiche pubbliche. Si rinvengono sostanzialmente tre modi di affrontare la tematica. Il primo modo, che si avvicina strettamente al polo sinistro del nostro continuum è quello di fare leva su un uso ristretto e delimitato di apprendimento al solo scopo di puntare i riflettori sulla dimensione epistemica dell'azione politica, sulla sfera della conoscenza in quanto tale. In questo caso l'apprendimento implica la possibilità di risolvere problemi e di scoprire nuove soluzioni grazie all'accrescimento delle conoscenze tecniche. Il cambiamento, come esito finale del processo di apprendimento, è sinonimo di perfezionamento e di innovazione. Il metodo opposto, identificato nella polarità destra del continuum, è quello di dilatare la nozione di apprendimento sino a considerarlo un prius, un passaggio obbligato per la comprensione del cambiamento e dell'azione medesima. L'attenzione viene rivolta alle cognizioni degli attori e al conseguente modellamento delle strategie individuali e collettive sulla base delle rappresentazioni soggettive. Il concetto di cambiamento si allontana da quello di innovazione, diventando spesso sinonimo di reiterazione. Il terzo modo, infine, a metà tra i due modelli, suggerisce convincentemente di guardare ai processi di apprendimento in maniera trasversale rispetto al fenomeno politico, tenendo conto che gli individui apprendono non solo sotto il profilo delle cognizioni, ma anche sotto il profilo degli assetti istituzionali entro cui si esplica la loro azione. Le informazioni in merito a specifici problemi vengono assimilate ed elaborate su uno spettro ampio di dimensioni calcolandone le ripercussioni sul contesto politico e sociale. Il mutamento è un fenomeno complesso che trae origine dal cammino passato della politica, nel corso del quale si è assistito alla sinergica mobilitazione di numerose costellazioni di fattori.

I tre passaggi non sono di poco conto e le loro estensioni non paiono ovvie nell'ambito del dibattito sulle determinanti del policy change.

Nella prima prospettiva si riflette l'interesse verso l'apprendimento orientato alla valutazione. Apprendere significa raccogliere e selezionare informazioni al fine di migliorare le proprie conoscenze e di risolvere più efficacemente $i$ problemi. Il cambiamento è di natura tecnica: gli individui accedono a nuovi dati per perfezionare la loro attività di sperimentazione e di misurazione. Secondo Wildavsky «nessun tipo di valutazione è possibile senza le informazioni adeguate» $(1979,235)$. A tale 
proposito acquista rilevanza la natura organizzativa del cambiamento. Gli individui entrano nelle gerarchie organizzative allo scopo di ottenere una maggiore quantità di dati e di convertirli in informazioni. L'accesso alle informazioni si traduce in una risorsa di potere che a sua volta facilita l'accumulazione dell'expertise (ibidem, 231). In questo primo modello appare dunque ridotta e precisamente delimitata l'area di applicazione delle dinamiche cognitive; le idee e gli assunti valoriali sono semplicemente filtri per il perfezionamento delle competenze acquisite e per la generazione di nuove soluzioni.

La prospettiva opposta viene compresa entro gli approcci post-moderni che mettono a fuoco il ruolo attivo non solo delle strategie degli attori (quindi dei comportamenti), ma, con un passaggio in più, delle cognizioni e delle rappresentazioni soggettive che modellano tali strategie d'azione. Il nesso azionestruttura si sposta a favore dell'azione e l'azione non è più scindibile dai quadri interpretativi che la originano. Il punto centrale diventa indagare intorno al modo in cui l'emergere di ogni soluzione è imputabile all'attività di elaborazione soggettiva degli attori. Le ricerche condotte in questo ambito indicano come il cambiamento assuma una natura ciclica e come i decisori si trovino di frequente intrappolati in situazioni bloccate. Secondo Schram le riforme del sistema di welfare hanno la funzione non tanto di risolvere problemi quanto di ricrearli, di replicare il contesto entro cui occorre intervenire $(1993,261)$. Le riforme riproducono le differenze sociali attraverso l'endemica esclusione delle categorie non protette a tutto vantaggio di quelle già sovvenzionate. Ciò alimenta il circolo vizioso provocando un'ulteriore emarginazione dei disagiati. Le popolazioni di riferimento (target populations) per la distribuzione dei sussidi vengono «costruite» socialmente a prescindere dalle esigenze reali (Schneider e Ingram 1993). Lo studio del comportamento dei decisori pubblici e del loro modo di percepire la realtà sociale, economica e politica è dunque una condizione preliminare alla rilevazione dell'influenza di questa realtà sul processo politico. L'ecologia delle cognizioni - come l'insieme delle specifiche modalità attraverso cui i decisori interpretano e attivano il loro rapporto con le istituzioni dell'ambiente socio-economico (il mercato, i gruppi d'interesse, i cittadini) e della rappresentanza politica (le strutture di governo) - attenua il problema della statuizione di rapporti causali certi tra i fenomeni e le loro cause, dimostrando l'esistenza di interrelazioni reciproche e spesso 
ambivalenti. L'applicazione esasperata della dimensione cognitiva conduce tuttavia a situazioni «virtuali» in cui ogni azione ha una derivazione cognitiva $e$ in cui dunque l'autonomia esplicativa della variabile apprendimento rispetto alle altre variabili rischia di erodersi.

Una prospettiva ancora diversa viene invece postulata dai modelli che occupano le posizioni centrali del continuum. È qui che si riscontrano significative aree d'intersezione con l'analisi politica e che dunque pare meritorio soffermarsi. L'apprendimento di policy viene qui considerato come indicatore dell'evoluzione complessiva della politica. Esso tuttavia non equivale alla scoperta della variabile indipendente che, da sola, è capace di spiegare il decorso delle politiche (Capano 1995): il suo uso non avviene in vacuum, bensì in interazione con altre dimensioni. La multicausalità è data dal riconoscimento del contemporaneo concorso di una molteplicità di fattori, tra cui anche i processi di evoluzione endogeni ai sottosistemi di policy (Ferrera $1993)^{12}$. In sintonia con il ragionamento inaugurato da Lowi (1964; 1972), le caratteristiche della politica possono trasformarsi da explanandum in explanans del processo politico influenzando direttamente la sfera del contesto politico-istituzionale circostante (Cotta 1989). Secondo Heclo (1974) gli studi tradizionali sulle politiche vanno infatti criticati non solo a causa del dominio imperante del potere come principale categoria esplicativa, bensì per l'abitudine invalsa di valutare l'impatto delle determinanti sul cambiamento in reciproca esclusione. L'affannosa ricerca della variabile indipendente, a monte di serrate catene causali, di volta in volta identificata nei fattori socioeconomici (crescita economica, andamento demografico, configurazione dei gruppi d'interesse) o nei fattori politico-istituzionali (forma di stato, sistema dei partiti, dinamica della competizione elettorale e configurazione degli apparati amministrativi), offusca la connessione tra le variabili a tutto discapito della possibilità di cogliere la complessità e la molteplicità dei fenomeni reali. Non solo i fattori condizionanti si intrecciano co-

12 L'aggettivo «multicausale» viene qui utilizzato nell'accezione proposta da Ferrera nell'ambito della ricostruzione delle politiche sociali nelle democrazie occidentali. L'autore mette a punto uno schema esplicativo multicausale che gli consente di combinare le «logiche contestuali, quelle istituzionali e quelle politico-processuali» (1993, 107) nella spiegazione dei processi di riforma sociale. In questo modo, egli suggerisce di ragionare sull'interazione tra «costellazioni di fattori» superando il determinismo insito nella ricerca di un'unica variabile esplicativa. 
stantemente ma ad essi si aggiunge un'altra famiglia di variabili legate all'eredità della politica stessa (policy inberitance). Le caratteristiche delle politiche sono espressione di stringenti legami con le esperienze passate, con il sentiero delle opportunità che ne ha scandito il decorso storico. Le innovazioni sono reazioni al retroterra di tecniche, metodi e linguaggi sviluppatisi nel passato. In questo senso, lo sviluppo delle riforme sociali può considerarsi come il tentativo di reagire alle condizioni dettate dalle leggi sui poveri.

L'analisi di Heclo trova assonanze più che fortunate nelle recenti teorie del mutamento istituzionale (David 1985 e 1992; Arthur 1989; North 1994). Secondo questa prospettiva, le ragioni dell'evoluzione e del ristagno dell'economia risultano strettamente dipendenti dalle specifiche combinazioni di vincoli e di opportunità che si sono formati nel passato (path dependency). Il potenziale di innovazione si trova in qualche modo «scritto geneticamente» nel cammino segnato dall'esperienza. Benché nel punto di emergenza delle policies si diano condizioni stocastiche, gli assetti iniziali manifestano un'elevata persistenza e una profonda inerzia organizzativa. Lo sviluppo istituzionale è dunque di natura incrementale, presentandosi come una sequenza di aggiustamenti locali successivi. L'evoluzione per apprendimento implica effetti di inerzia (lock-in effects: Arthur 1989) che condizionano «dal di dentro» le future alternative di sviluppo. Si producono incentivi che incoraggiano gli individui ad agire in modo prevalentemente adattivo e ripetitivo in «sentieri di sviluppo condizionato» (North 1994, 150) che tendono a bloccare le grandi deviazioni e i cambiamenti repentini. In un modello di spiegazione «genealogico» (di contro al modello esplicativo teleologico, caro agli economisti neoclassici) le politiche sono «portatrici di storia» (carriers of bistory: David 1992); nella loro forma attuale si leggono le combinazioni di successo del passato ${ }^{13}$.

La rilevanza di un'analisi interdisciplinare condotta su dimensioni temporali e spaziali multiple viene evidenziata a più riprese nello studio dei processi di imitazione e di diffusione dei programmi, descritti da Rose e da Haas. La circolazione e

${ }^{13}$ È già stato da più parti sottolineato come gli assetti istituzionali si sviluppino per «stratificazioni successive»: il modello originario (Panebianco 1982; Zan 1985) lascia le sue impronte in ogni sequenza successiva. Per ulteriori approfondimenti rimandiamo in particolare a Freddi (1989). 
l'assimilazione delle nuove idee sono funzione del loro grado di permeabilità rispetto ad un ampio spettro di interessi e di appartenenze. I programmi esprimono «aggregazioni di interessi che accomunano funzionari pubblici, rappresentanti dei gruppi di interesse, professionisti e clienti» (Rose 1991, 7). La presenza di circuiti di esperti che agiscono a livello internazionale, fornendo comuni quadri interpretativi sul come trattare le variabili macroeconomiche a governi di diverso orientamento ideologico, mostra la rilevanza della conoscenza tecnica come variabile in grado di integrare la conoscenza politica (Haas 1992).

Anche Sabatier e Jenkins-Smith (1993) guardano alla cumulazione evolutiva delle dinamiche interne ai networks di policy come ad uno dei fattori che concorrono a riorientare le politiche. Il cambiamento, secondo questi autori, è infatti un fenomeno complesso, risultato degli slittamenti nelle condizioni socioeconomiche esterne, nella coalizione di governo e nel sistema di regole formali che definiscono il contesto istituzionale. I fattori micro direttamente attinenti alle caratteristiche della politica si combinano con fattori macro di natura strutturale.

La sinergica attivazione di «famiglie di variabili», in assenza di rigidi rapporti di determinazione causale, viene postulata infine da Hall (1989) nel tentativo di dare ragione dell'adozione delle politiche keynesiane da parte degli Stati Uniti e dei paesi europei. Il processo attraverso cui le nuove soluzioni s'impongono sulle vecchie, dando inizio alla sostituzione del paradigma di policy dominante, viene filtrato da tre clusters di fattori: i fattori direttamente legati ai contenuti dei programmi (economic viability), i fattori di natura politica (political viability) e i fattori amministrativi di natura strutturale (admnistrative viability).

In sintesi, il tentativo principale di queste teorie in relazione allo studio del cambiamento è quello di evidenziare la complessità dell'azione sociale e di proporre chiavi di lettura multiple che intrecciano in modo interdipendente la configurazione degli assetti istituzionali con gli interessi degli individui che ne fanno parte, le loro identità programmatico-ideologiche e la loro capacità di percepire e di risolvere problemi. Secondo questi autori è dunque di minore utilità selezionare un solo gruppo di variabili, ad esempio socio-economiche (Dye 1966) oppure politiche (Alber 1986; Castles 1982), chiedendosi quali siano quelle che nell'intero decorso evolutivo della politica dominano sulle altre e che determinano la nascita di soluzioni innovative (pensiamo alla disputa sul does politics matter?). È più realistico 
ipotizzare l'esistenza di combinazioni di fattori che in un dato momento esercitano effetti e nello stesso tempo sono influenzati dall'evoluzione della politica. Solo la ricostruzione empirica di alcune sequenze del cammino della policy attraverso l'attenta disamina delle sue caratteristiche «locali» e del modo attraverso cui queste caratteristiche sono plasmate dagli assetti istituzionali circostanti può facilitare l'analisi e la comprensione. Gli individui apprendono ad interagire con le istituzioni nel senso che, pur all'interno di un campo delimitato di strategie possibili, essi contrattano continuamente nuovi spazi per l'azione (Friedberg 1994). L'apprendimento è dunque un fenomeno complesso: al tempo stesso sociale, politico e di policy.

Apprendimento sociale, politico, di policy e il problema del rapporto tra azione e struttura

Nell'ambito della prospettiva multicausale sopra delineata è ora possibile compiere un passaggio ulteriore: costruire una tipologia dell'apprendimento che integri (specificandola) la definizione dei fattori che concorrono a spiegare il cambiamento politico. Il nostro obiettivo non è tanto quello di proporre un nuovo modello esplicativo, quanto di studiare il cambiamento dal punto di vista delle strategie degli attori (rispetto alle strutture) o, ancora meglio, dal punto di vista dell'interazione tra individui e istituzioni, recuperando in questo modo il contributo specifico degli studi sino ad ora presentati. Il problema del raccordo «agency-structure» (Hall e Taylor 1994) è dunque il nostro quesito cruciale. Identifichiamo tre tipi di apprendimento: sociale, politico e di policy, in analogia con i tre principali clusters di fattori a cui solitamente viene attribuito il mutamento politico. Essi riguardano, rispettivamente, il rapporto tra gli attori e il contesto economico e sociale (le istituzioni del mercato e della rappresentanza funzionale) nell'ambito della formulazione dei problemi di policy; il rapporto tra i decisori e le istituzioni della rappresentanza politica, nell'ambito dell'attività di ricerca del consenso; e le interazioni tra le strategie degli attori nei network di policy in occasione della messa a punto di strumenti e di metodi per attuare le decisioni. I tre tipi, che qui separiamo per fini euristici, sono circolarmente collegati ${ }^{14}$; la mo-

${ }^{14}$ Ringrazio Peter Hall per avermi invitato a riflettere su questo punto. 
dificazione degli strumenti con cui risolvere i problemi si ripercuote sulla successiva ridefinizione del problema. Si sviluppano effetti cumulativi: ogni sequenza diventa feedback per quella successiva. I processi di apprendimento rappresentano infatti un tipo particolare di cambiamento che avviene per evoluzione incrementale, attraverso spostamenti locali reiterati ${ }^{15}$.

Per apprendimento sociale intendiamo il processo attraverso cui gli attori giungono a definire ciò che è per loro conveniente sulla base dei vincoli e delle opportunità dell'ambiente esterno. L'attenzione è rivolta al modo in cui prendono vita le aggregazioni degli interessi categoriali. La capacità di organizzazione e di pressione dei gruppi, potenzialmente in grado di dettare le riforme sociali, trae origine dal modo in cui gli individui riescono a reagire all'incertezza ambientale e a ricavarsi nuove nicchie per l'azione. Le condizioni dell'ambiente economico (la disponibilità di risorse) e le regole della rappresentanza definiscono il contesto entro cui gli individui possono (o non possono) promuovere aggregazioni collettive in grado di formulare legittimamente la domanda sociale. Il grado di sviluppo dei gruppi d'interesse è anche funzione dell'esigenza di difendere sistemi di credenza condivisi. Gli esperimenti di deregolazione occorsi in Inghilterra alla fine degli anni settanta rappresentano un esempio interessante di apprendimento sociale. L'abbandono delle politiche keynesiane e di un modello interventista dell'azione statale ha implicato la mobilitazione di numerosi attori politici e sociali (Hall 1993). L'elezione di Margaret Thatcher, lo spostamento dell'autorità del Tesoro alla banca centrale, l'intensificarsi del dibattito pubblico su temi economici e finanziari, hanno permesso una lettura dell'ambiente favorevole alla sostituzione del paradigma di policy dominante e all'innovazione delle regole del gioco macroeconomico. Nella categoria dell'apprendimento sociale rientrano dunque una molteplicità di attori di diversa provenienza: ai gruppi d'interesse si associano le aggregazioni spontanee, i politici e i liberi cittadini. L'oggetto dell'apprendimento può variare dalla formulazione di nuovi programmi e dalla progettazione di iniziative di voice, alla semplice esplicitazione di un senso di appartenenza. La dinamica del

15 L'apprendimento in qualche misura sottende una teoria dell'efficienza della storia, poiché si danno incrementi positivi (in senso tecnico non valutativo) da un ciclo all'altro (Unger 1987; March 1988). 
processo può essere incrementale oppure improntata a discontinuità, in periodi, ad esempio, di mobilitazione sociale.

Una volta formulata, la domanda sociale deve tradursi in domanda politica attraverso il meccanismo del voto. Il processo attraverso cui gli attori politici apprendono quale correlazione vi sia (e come sia manipolabile) tra atti decisionali e spostamenti nelle quote di consenso (preferenze elettorali) definisce l'apprendimento politico. Il nesso decisione/consenso è al centro di questo processo. Gli attori politici giocano un ruolo rilevante (quindi politics does matter), prima ancora perché rappresentanti di un partito con una determinata identità ideologico-programmatica, per la capacità di seguire, o meglio, di anticipare le preferenze degli elettori. L'apprendimento politico si riflette in una lettura proattiva dell'ambiente. Nel caso inglese il dibattito pubblico sulle politiche economiche balza velocemente nell'arena politica, diventando l'issue più saliente della competizione elettorale e dell'agenda governativa. La sostituzione del tema della disoccupazione (centrale nel paradigma keynesiano) con quello dell'inflazione diventa un'arma potente, soprattutto dal punto di vista della simbologia politica, per sfidare il partito laburista. Margaret Thatcher in qualche misura riesce a volgere a proprio favore il desiderio di cambiamento diffuso nella società. $\mathrm{Si}$ assiste ad un'immediata politicizzazione delle tematiche economiche che vengono affrontate nei termini delle quote di consenso associate. Nel medesimo periodo, anche la precipitosa ritirata dai propri intenti programmatici da parte dei partiti socialisti francese e spagnolo e della conseguente adozione di politiche del rigore di matrice conservatrice e liberista (Pappalardo 1991 e 1992) testimonia assai convincentemente della capacità di apprendimento strategico degli attori politici. La relativa neutralizzazione degli imperativi dell'appartenenza mostra come i vincoli contestuali non risultino separabili dalle reinterpretazioni degli attori. Vi è infatti uno stretto legame tra azione individuale e contesto istituzionale: è vero che sono $i$ soggetti individuali (i politici e i burocrati) che apprendono, ma il fenomeno è riconoscibile per il fatto che le istituzioni imparano ad accogliere determinati quadri interpretativi entro cui si rinviene l'insieme delle norme formali e informali che governano l'azione politica (Vassallo 1994). In questa ottica diventa labile e sfumato il confine tra fattori micro e fattori macro: l'azione individuale è filtrata dal contesto istituzionale e le istituzioni altro non sono che la cristallizzazione dell'esperienza collettiva. Esse 
costituiscono un campo di regole e di norme per l'azione, costantemente rimodellate dalle percezioni degli individui sul loro uso e sulla loro utilità. La dinamica dell'apprendimento politico è infatti informata alla negoziazione e alla mediazione, data l'esigenza di composizione degli interessi divergenti.

L'implementazione delle decisioni formulate nella sfera politico-istituzionale richiede, infine, che gli individui apprendano a risolvere problemi. L'apprendimento di policy è dunque il processo attraverso cui gli attori nei sottosistemi di policy acquisiscono nuove informazioni al fine di modificare gli strumenti esistenti e di creare nuove tecniche per realizzare gli obiettivi attesi. Nel caso britannico la sostituzione degli strumenti keynesiani (politiche dei redditi, politiche fiscali, limiti ai prestiti bancari) con strumenti associati a politiche deregolative (prevalentemente connessi alla manipolazione dei tassi d'interesse) costituisce l'ultimo tassello del mosaico monetarista.

Nei networks che si costituiscono intorno all'implementazione dei programmi di policy l'interazione continua tra gli attori e lo sviluppo di logiche cooperative e di comportamenti solidaristici a prescindere dalle appartenenze originarie crea un clima favorevole per la generazione di innovazioni e di sperimentazioni. Gli strumenti e i metodi contenuti nei programmi insieme all'acquisizione di un linguaggio comune sono l'oggetto dell'apprendimento; la natura del processo è per lo più incrementale, caratterizzata da effetti cumulativi e da aggiustamenti sequenziali.

Riportando sinteticamente le caratteristiche principali dei tre tipi di apprendimento in uno schema complessivo (fig. 2), si evince come le maggiori differenze ineriscano ai soggetti coinvolti nel processo di apprendimento, rilevandosi di contro distinzioni sfumate in relazione all'oggetto e alla dinamica del processo. L'apprendimento sociale coinvolge i soggetti solitamente esclusi dal circuito politico-formale della rappresentanza, mentre l'apprendimento politico mobilita direttamente i decisori pubblici. Nel caso dell'apprendimento di policy, infine, la partecipazione si allarga sino a comprendere soggetti di diversa provenienza, superando la linea divisoria tra canali di accesso formali e informali. I tipi di apprendimento indicano pertanto livelli di analisi differenti, entro $\mathrm{i}$ quali si verificano mutamenti e aggiustamenti nel cammino evolutivo delle politiche. Occorre tuttavia ricordare, come abbiamo già accennato in precedenza, che $\mathrm{i}$ confini tra le tre colonne sono piuttosto sfumati; la circo- 
tipi di apprendimento

\begin{tabular}{|c|c|c|c|}
\hline & $\begin{array}{l}\text { apprendimento } \\
\text { sociale }\end{array}$ & $\begin{array}{l}\text { apprendimento } \\
\text { politico }\end{array}$ & $\begin{array}{l}\text { apprendimento di } \\
\text { policy }\end{array}$ \\
\hline soggetti & $\begin{array}{l}\text { individui, gruppi, } \\
\text { movimenti, } \\
\text { comunità }\end{array}$ & $\begin{array}{l}\text { istituzioni } \\
\text { politiche: politici } \\
\text { e burocrati* }\end{array}$ & $\begin{array}{l}\text { attori del network: } \\
\text { politici, esperti, } \\
\text { burocrati, } \\
\text { rappresentanti dei } \\
\text { gruppi }\end{array}$ \\
\hline oggetto & $\begin{array}{l}\text { progettazione di } \\
\text { programmi e } \\
\text { iniziative di } \\
\text { mobilitazione }\end{array}$ & $\begin{array}{l}\text { programmi } \\
\text { pubblici: leggi e } \\
\text { documenti }\end{array}$ & $\begin{array}{l}\text { strumenti, metodi } \\
\text { e linguaggi }\end{array}$ \\
\hline $\begin{array}{l}\text { dinamica: } \\
\text { natura } \\
\text { dell'apprendimento }\end{array}$ & $\begin{array}{l}\text { incrementalismo } \\
\text { e/o mobilitazione }\end{array}$ & $\begin{array}{l}\text { mediazione e } \\
\text { negoziazione }\end{array}$ & $\begin{array}{l}\text { adattamento e } \\
\text { incrementalismo }\end{array}$ \\
\hline
\end{tabular}

FIG. 2. Schema di sintesi sui tipi di apprendimento.

* Consideriamo del tutto superata la proposizione formale secondo la quale i burocrati rappresenterebbero un apparato neutrale e imparziale, preposto all'esclusiva esecuzione delle leggi. È già stato ampiamente dimostrato come, a disconferma delle ipotesi assiologiche della teoria democratica, non si dia una rigida distinzione tra politica e amministrazione e come i burocrati partecipino attivamente al processo di formulazione legislativa (Putnam 1973; Freddi 1989).

larità del processo crea numerose aree di giustapposizione. La scoperta di nuove soluzioni retroagisce sull'originaria costellazione degli interessi promuovendo modificazioni e ristrutturazioni: si sviluppa una funzione di apprendimento che scaturisce dagli effetti lasciati dalla politica stessa. In riferimento alle politiche di deregolazione in Gran Bretagna, ad esempio, alcuni studi recenti mettono in luce come dopo i primi anni del governo Thatcher in realtà si assista ad un ritorno delle politiche keynesiane, a dimostrazione della rilevanza dell'eredità della politica (Garrett 1993).

In questo senso, si può sostenere che la policy ha una dimensione cognitiva, una conoscenza che la società riesce a produrre su se stessa attraverso fenomeni di perfezionamento e di degrado successivi. 


\section{Prospettive di ricerca}

Al termine delle nostre riflessioni vorremmo proporre, più che un bilancio conclusivo, un'anticipazione delle possibili direzioni di ricerca, tenendo conto dei numerosi interrogativi lasciati aperti dalla letteratura e del senso di smarrimento che la presentazione sintetica di suggestioni teoriche eterogenee può aver generato. Vi è una questione cruciale da cui qualsiasi tipo di indagine sul policy learning non può sottrarsi ed è quella relativa alla definizione operativa della variabile apprendimento. Come argomenta Bateson «non c'è dubbio che la parola "apprendimento" denoti un cambiamento di qualche tipo: dire quale tipo di cambiamento è una faccenda delicata» (1976, 307). Qual è dunque il discrimine tra cambiamento e apprendimento? $\grave{E}$ possibile isolare la variabile apprendimento al fine di misurarne gli effetti? E $\mathrm{E}$ possibile costruire un test che permetta di verificare se in un dato momento dell'evoluzione della politica le dinamiche cognitive hanno contato di più rispetto alle dinamiche degli interessi?

La questione ha ovviamente natura squisitamente empirica e poco si addice al grand theorizing. Le poche ricerche sino ad ora condotte (ad esempio May 1992) lasciano invero insoddisfatti. Almeno da due punti di vista: in termini metodologici, si assiste ad un uso «selvaggio» di tecniche quantitative in tangibile dissonanza con la natura qualitativa di questa letteratura; in termini sostantivi, si constata che i risultati ottenuti, sulla base peraltro di fonti secondarie, sono spesso deludenti rispetto alle aspettative, dando la fastidiosa impressione di indugiare in una mera operazione cosmetica: la sovrapposizione dell'etichetta «apprendimento» ad eventi interpretabili con altri modelli.

D'altra parte, occorre al contempo ridimensionare le aspettative, riconoscendo che sarebbe un errore esasperare l'antinomia tra cambiamento e apprendimento, data la natura dilemmatica del rapporto che lega i due termini. L'apprendimento è infatti una metafora (Gherardi 1994), un modo per studiare il cambiamento gettando luce sul retroterra di idee, norme e valori che informano le strategie degli attori, e un modo di vedere le istituzioni come se fossero sistemi che apprendono. Le metafore hanno funzioni ben delimitate: attraverso il meccanismo dell'analogia aiutano a scoprire aspetti inesplorati dei fenomeni osservati oppure a guardare con lenti diverse i medesimi fenomeni. La possibilità di esplorare il cammino evolutivo delle po- 
litiche da un punto di osservazione differente è già di per sé significativa.

In termini metodologici è possibile nondimeno abbozzare alcuni suggerimenti. Secondo Sabatier e Jenkins-Smith, l'apprendimento è funzione di almeno tre variabili: il livello del conflitto esistente tra le diverse coalizioni degli interessi nei network di policy; la trattabilità analitica del problema; e la presenza di gruppi di esperti. Il livello del conflitto esprime il «grado di incompatibilità tra i sistemi di credenza delle diverse coalizioni» $(1993,49)$ : esso nasce quando le nuove direzioni intraprese dalla policy scalfiscono i valori dominanti che governano le strategie delle élites. Il processo di apprendimento è favorito da un livello intermedio di discussione, in cui i rappresentanti dei diversi interessi in competizione lottano per l'imposizione dei propri sistemi di credenza. Secondo gli autori, in una situazione di conflittualità controllata è più probabile che ogni gruppo disponga delle risorse tecniche necessarie per prendere parte al dibattito. Il secondo indicatore di un processo di cambiamento, endogeno al sotto-settore di policy, si riferisce al grado di complessità del problema, riconducibile alla possibilità di raccolta e di trattamento dei dati e di applicazione di modelli interpretativi chiari e riconoscibili. L'apprendimento risulta ostacolato quando il fuoco dell'analisi, la politica al centro del conflitto, risulta di difficile codificazione. L'esistenza di dati certi e di teorie interpretative condivise agevola l'attivo coinvolgimento dei partecipanti al network. Il terzo indicatore, infine, è di natura strettamente epistemica e riguarda il grado di apertura del network rispetto ai gruppi esterni di professionisti, che dispongono di conoscenza e di expertise in relazione al proble$\mathrm{ma}$ in questione. Distinguendo tra «circoli chiusi» e «circoli aperti» a seconda della natura dell'accesso, i due studiosi depongono a favore di network sufficientemente ampi e prestigiosi, in grado di attirare esperti di varia provenienza.

In questa prospettiva, dunque, l'operazionalizzazione del grado di apprendimento avviene prevalentemente attraverso la rilevazione del livello di acquisizione e di disponibilità di informazioni tecniche. Maggiori sono le conoscenze a disposizione, più frequenti diventano gli scambi di informazioni, più probabile è la possibilità di imprimere nuovi corsi d'azione alle politiche e di generare nuove soluzioni per risolvere problemi complessi.

Questi indicatori possono tuttavia essere integrati da due al- 
tri fattori che permettono di isolare ulteriormente la variabile apprendimento: il surclassamento della logica dell'appartenenza $e$ la natura dell'innovazione ${ }^{16}$.

Il primo fattore si riferisce alla relativa estraneità delle strategie degli attori rispetto a un diretto calcolo di potere. La valutazione dell'influenza dell'affiliazione a gruppi e a partiti sulle strategie di azione dei partecipanti ai network di policy permette di verificare l'eventuale scavalcamento delle identità ideologico-programmatiche originarie e l'apertura di tavoli di discussione comuni. Soprattutto a livello decentrato risulta proficuo esaminare l'impatto dell'avvicendarsi dei governi locali sulla formulazione e sull'attuazione dei programmi.

Il secondo indicatore rimanda invece alla configurazione dell'innovazione, alla dinamica con cui il cammino evolutivo della politica subisce interruzioni e modificazioni. Come abbiamo già illustrato in precedenza, l'attivazione dei processi di apprendimento avviene attraverso spostamenti graduali e continuativi: le innovazioni si presentano come insieme di effetti cumulativi successivi e non come mutamenti radicali (o come shock). Il processo attraverso cui i nuovi stimoli vengono adattati al contesto di pratiche esistenti, e quindi a loro volta costantemente «modificati», costituisce l'essenza dell'apprendimento.

Non solo, quindi, la ricostruzione dei processi di apprendimento rafforza la convinzione teorica che l'interazione strategica degli individui nei sottosistemi di policy è prodotto e allo stesso tempo causa del processo politico, dando nuova risonanza al dibattito sulle determinanti del cambiamento politico; ma essa stessa rappresenta un'occasione empirica privilegiata per progettare nuovi disegni di ricerca e per intraprendere indagini più vicine alle cognizioni e alle interpretazioni degli attori, a cui forse rischiamo di essere meno avvezzi.

${ }^{16}$ Chi scrive è attualmente impegnata in una ricerca comparata sull'apprendimento istituzionale nell'ambito delle politiche del lavoro italiane e tedesche. Nel caso tedesco, dopo lo «shock dell'unificazione», per rispondere alla dilagante disoccupazione dei nuovi Länder, si è assistito alla creazione di una serie di nuove disposizioni per la tutela dei senza lavoro sulla base di correttivi e di modificazioni al quadro delle misure esistenti. Il processo è avvenuto per passaggi sequenziali successivi: gli attori decisionali hanno gradualmente «imparato» a correggere i programmi ordinari, scoprendo nel quadro dei vincoli esistenti nuovi interstizi per l'azione. 


\section{Riferimenti bibliografici}

Alber, J. (1986), Dalla carità allo stato sociale, Bologna, Il Mulino (ed. orig. Vom Armenhaus zum Woblfabrs Taat, Frankfurt, Campus Verlag, 1982).

Argyris, C. e D. Schön (1978), Organizational Learning. A Theory of Action Perspective, Reading MA, Addison Wesley.

Arthur, B.W. (1989), Competing Technologies, Increasing Returns and Lock-in by Historical Events, in «Economic Journal», 99, pp. 116.

Bennett, C.J. e M. Howlett (1992), The Lessons of Learning: Reconciling Theories of Policy Learning and Policy Change, in «Policy Sciences», XXV, pp. 275-294.

Bateson, G. (1976), Verso un'ecologia della mente, Milano, Adelphi.

Braybrooke, D. e C.E. Lindblom (1970), A Strategy of Decision. Policy Evaluations as a Social Process, New York, The Free Press.

Capano, G. (1995), Il policy change: tra politica delle idee e politica degli interessi, in «Teoria Politica», XI, 1, pp. 133-165.

Castles, F.G. (1982), The Impact of Parties: Politics and Policies in Democratic Capitalist States, London, Sage.

Cotta, M. (1989), Il contributo dei «policy studies» alla scienza politica contemporanea, in A. Panebianco (a cura di), L'analisi della politica, Bologna, Il Mulino, pp. 517-541.

- (1994), Il governo di partito in Italia. Crisi e trasformazione dell'assetto tradizionale, in M. Caciagli, F. Cazzola, L. Morlino e S. Passigli (a cura di), L'Italia fra crisi e transizione, Bari, Laterza, pp. 119-139.

Crozier, M. (1978), Il fenomeno burocratico, Milano, Etas Libri (ed. orig. Le phénomène bureaucratique, Paris, Ed. du Seuil, 1963).

David, P. (1985), Clio and the Economics of QWERTY, in «American Economic Review», LXXV, pp. 332-337.

- (1992), Why Are Institutions the «Carriers of History»?, Discussion Paper, Stanford University.

Deutsch, K.W. (1963), The Nerves of Government, New York, The Free Press; trad. it. I nervi del potere, Milano, Etas Kompass, 1972.

Dobuzinskis, L. (1992), Modernist and Postmodernist Metaphors of the Policy Process: Control and Stability vs. Chaos and Reflexive Understanding, in «Policy Sciences», XXV, pp. 355-380.

Dye, T.R. (1966), Politics, Economics and the Public: Policy Outcomes in the American States, Chicago, Rand McNally.

Etheredge, L.S. e J. Short (1983), Thinking about Government Learning, in «Journal of Management Studies», XX, 1, pp. 41-58.

Ferrera, M. (1993), Modelli di solidarietà. Politica e riforme sociali nelle democrazie, Bologna, Il Mulino.

Forester, J. (1982), The Policy Analysis-Critical Affair: Wildavsky and 
Habermas as Bedfellows?, in «Journal of Public Policy», 2, pp. 145-163.

Freddi, G. (a cura di) (1989), Scienza dell'amministrazione e politiche pubbliche, Roma, La Nuova Italia Scientifica.

Friedberg, E. (1994), Il potere e la regola. Dinamiche dell'azione organizzativa, Milano, Etas Libri; (ed. orig. Le pouvoir et la règle. Dynamiques de l'action organisée, Paris, Ed. du Seuil, 1993).

Garrett, J. (1993), The Politics of Structural Change: Swedish Social Democracy and Thatcherism in Comparative Perspective, in «Comparative Political Studies», XXV, 4, pp. 521-547.

Gherardi, S. (1994), Imparare a decidere, in D. Demetrio et al. (a cura di), Apprendere nelle organizzazioni, Roma, La Nuova Italia Scientifica, pp. 161-208.

Gualmini, E. (1995), Apprendimento (e politiche pubbliche), in G. Capano e M. Giuliani (a cura di), Dizionario delle politiche pubbliche, Roma, La Nuova Italia Scientifica, in corso di pubblicazione.

Haas, P. (1992), Introduction: Epistemic Communities and International Policy Coordination, in «International Organization», XLVI, pp. $1-35$.

Hall, P.A. (1986), Governing the Economy. The Politics of State Intervention in Britain and in France, Cambridge, Polity Press.

- (1993), Policy Paradigms, Social Learning and the State. The Case of Economic Policymaking in Britain, in «Comparative Politics», XXV, 3, pp. 275-296.

Hall, P.A. (a cura di), (1989), The Political Power of Economic Ideas: Keynesism Across Countries, Princeton, Princeton University Press.

Hall, P.H. e P.C.R. Taylor (1994), Political Science and Four New Institutionalisms, Discussion Paper for the Annual Meeting of the American Political Science Association.

Heclo, H. (1974), Modern Social Politics in Britain and in Sweden, New York, Yale University Press.

Hempel, C.G. (1968), Filosofia delle scienze naturali, Bologna, Il Mulino.

Kingdon, J.W. (1984), Agendas, Alternatives and Public Policies, Boston, Little Brown \& Co.

Landau, M. (1973), On the Concept of a Self-Correcting Organization, in «Public Administration Review», 3, pp. 533-543.

Lanzara, G.F. (1993), Capacità negativa. Competenza progettuale e modelli di intervento nelle organizzazioni, Bologna, Il Mulino.

- (1994), L'apprendimento organizzativo, di prossima pubblicazione in G. Costa e R. Nacamulli (a cura di), Manuale di Organizzazione.

Lowi, T.J. (1964), American Business, Public Policy, Case-Studies and Political Theory, in «World Politics», 16, pp. 677-715.

- (1972), Four Systems of Policy, Politics and Choice, in «Public Administration Review», 4, pp. 298-310. 
Majone, G. (1987), Forme di controllo e apprendimento istituzionale, in «Rivista Trimestrale di Scienza dell'Amministrazione», 1, pp. 25-38.

- (1989), Evidence, Argument and Persuasion in the Policy Process, New Haven and London, Yale University Press.

March, J.G. (1988), Variable Risk Preferences and Adaptive Aspirations, in «Journal of Economic Behavior and Organization», 9, pp. 5-24.

Maturana, H.R. e Varela (1987), The Tree of Knowledge, Boston, Shambala.

May, P.J. (1992), Policy Learning and Failure, in «Journal of Public Policy», XII, 1, pp. 331-354.

North, C.D. (1994), Istituzioni, cambiamento istituzionale, evoluzione dell'economia, Bologna, Il Mulino (Institutions, Institutional Change and Economic Performance, Cambridge, Cambridge Univ. Press, 1990).

Panebianco, A. (1982), Modelli di partito. Organizzazione e potere nei partiti politici, Bologna, Il Mulino.

Pappalardo, A. (1991), Austerità conservatrice e rigore socialista: che differenza? I, in «Rivista Italiana di Scienza della Politica», XXI, 1, pp. 33-90.

- (1992), Il rigore socialista: vincolo o scelta?, in M. Calise (a cura di), Come cambiano i partiti, Bologna, Il Mulino, pp. 147-167.

Pierson, P. (1993), When Effect Becomes Cause. Policy Feedback and Political Change, in «World Politics», XLV, 595-628.

Putnam, R. (1973), Atteggiamenti politici dell'alta burocrazia nell'Europa occidentale, in «Rivista Italiana di Scienza Politica», III, 1, pp. 145-186.

Regonini, G. (1993), Il principe e il povero. Politiche istituzionali ed economiche negli anni ottanta, in «Stato e Mercato», 3, pp. 361403.

- (1994), Partiti, reti, giochi, politiche pubbliche, in M. Caciagli, F. Cazzola, L. Morlino e S. Passigli (1994), L'Italia fra crisi e transizione, Bari, Laterza, pp. 163-189.

Rice, R.E. e E.H. Rogers (1980), Reinvention in the Innovation Process, in «Knowledge», 3, pp. 499-514.

Rose, R. (1991), What is Lesson-Drawing?, in «Journal of Public Policy», II, 1, pp. 3-30.

Rosenau, P. (1993), Anticipating a Post-Modern Policy Current?, in «Policy Currents», 2, pp. 1-4.

Sabatier, P.A. e H.C. Jenkins-Smith (1993), Policy Change and Learning. An Advocacy Coalition Approach, Colorado, Westview Press.

Sartori, G. (1980), La politica. Logica e metodo in scienze sociali, Milano, SugarCo.

Schneider, A. e H. Ingram (1991), Systematically Pincbing Ideas: A 
Comparative Approach to Policy Design, in «Journal of Public Policy», I, 8, pp. 61-80.

- (1993), Social Construction of Target Populations: Implications for Politics and Policy, in «American Political Science Review», 2, pp. 334-347.

Schram, S.F. (1993), Postmodern Policy Analysis: Discourse and Identity in Welfare Policy, in «Policy Sciences», XXVI, pp. 249-270.

Simon, H. (1967), Il comportamento amministrativo, Bologna, Il Mulino (ed. orig. Administrative Behaviour, New York, MacMillan, 1947)

Vassallo, S. (1994), Il governo di partito in Italia (1943-1993), Bologna, Il Mulino.

Weir, M. e T. Skocpol (1985), State Structures and the Possibilities of Keynesian Responses to the Great Depression in Sweden, Britain and the United States, in P. Evans, D. Rueschemeyer e T. Skocpol (a cura di), Bringing the State Back In, New York, Cambridge University Press, pp. 107-168.

Weiss, J. (1989), The Power of Problem Definition: the Case of Government Paperwork, in «Policy Sciences», XXII, pp. 97-121.

Wildavsky, A. (1979), Speaking Truth to Power. The Art and Craft of Policy Analysis, New Brunswick, Transaction Inc.

Unger, R.M. (1987), False Necessities. Anti-Necessitarian Social Theory in the Service of Radical Democracy, Cambridge, Cambridge University Press.

Zan, S. (1985), L'analisi longitudinale delle organizzazioni, in «Annali di Storia dell'Impresa», 1, pp. 59-122. 